\title{
Framing effects on metacognitive monitoring and control
}

\author{
BRIDGID FINN \\ Columbia University, New York, New York
}

\begin{abstract}
Three experiments explored the contribution of framing effects on metamemory judgments. In Experiment 1, participants studied word pairs. After each presentation, they made an immediate judgment of learning (JOL), framed in terms of either remembering or forgetting. In the remember frame, participants made judgments about how likely it was that they would remember each pair on the upcoming test. In the forget frame, participants made judgments about how likely it was that they would forget each pair. Confidence differed as a result of the frame. Forget frame JOLs, equated to the remember frame JOL scale by a 1-judgment conversion, were lower and demonstrated a smaller overconfidence bias than did remember frame JOLs. When judgments were made at a delay, framing effects did not occur. In Experiment 2, people chose to restudy more items when choices were made within a forget frame. In Experiment 3, people studied Spanish-English vocabulary pairs ranging in difficulty. The framing effect was replicated with judgments and choices. Moreover, forget frame participants included more easy and medium items to restudy. These results demonstrated the important consequences of framing effects on assessment and control of study.
\end{abstract}

Although people are fairly accurate at assessing how well they have learned something, much research has shown that people's metacognitive judgments about their memory can be miscalibrated (Benjamin, Bjork, \& Schwartz, 1998; Koriat, 1997; Koriat, Sheffer, \& Ma'ayan, 2002; Metcalfe, 1998; Zechmeister \& Shaughnessy, 1980). For example, people's initial judgments of learning (JOLs) about how much they think that they will remember on an upcoming test typically show an overconfidence bias in which, on average, the judgments are higher than subsequent test performance (Koriat, Lichtenstein, \& Fischhoff, 1980; Lichtenstein, Fischhoff, \& Phillips, 1982; Metcalfe, 1998). People have been shown to be so certain in their incorrect answers that they are even willing to bet money in the belief that they are correct (Fischhoff, Slovic, \& Lichtenstein, 1977).

Judgment accuracy is also thought to have importance consequences for how people control their own learning. For example, an overconfident student may stop studying before actually mastering the material, resulting in a poor test score. As Nelson and Dunlosky (1991, p. 267) said, "The accuracy of JOLs is critical because if the JOLs are inaccurate, the allocation of subsequent study time will correspondingly be less than optimal." Recently, Metcalfe and Finn (2008) provided evidence that people's metacognitive judgments are linked directly to their choices for restudy, supporting the long held view that faulty metacognitive judgments can unfavorably affect study control (Benjamin et al., 1998; Dunlosky \& Hertzog, 1998; Koriat, 2002; Mazzoni \& Cornoldi, 1993;
Metcalfe, 2002; Nelson \& Dunlosky, 1991; Pressley \& Ghatala, 1990; Thiede, 1999). Metcalfe and Finn showed that when people's JOLs were manipulated independently of their recall performance, study choices were influenced by the judgment rather than by the performance. When the judgments were biased, the study choices reflected the same pattern. These results demonstrated a direct link between metacognitive monitoring and control of learning and underscored the importance of judgment accuracy for achieving effective self-guided learning.

Metacognitive overconfidence most likely arises through the use of memory-based processing heuristics, such as an evaluation of the fluency of information retrieved or cue or domain familiarity, that become available when one is making a judgment (Glenberg, Wilkinson, \& Epstein, 1982; Koriat, 1993; Koriat \& Bjork, 2006; Metcalfe, 1998; Metcalfe, Schwartz, \& Joaquim, 1993; Reder, 1987, 1988; Tversky \& Kahneman, 1974). According to Koriat et al. (1980), overconfidence occurs because people rely primarily on information that is consistent with the answer they have chosen and tend to neglect contradictory information. Of the various debiasing techniques that have been explored in an effort to reduce the overconfidence bias (Lichtenstein \& Fischhoff, 1980; Yates, Veinott, \& Patalano, 2003), one of the most successful techniques has been to ask people to change the way they make their judgments by generating counterfactual evidence for the answer they have just given (Hirt, Kardes, \& Markman, 2004; Hirt \& Markman, 1995; Hoch, 1985; Koehler, 1991; Koriat et al., 1980; Maki, 1998). Koriat et al. (1980) found improvements in the ac-

B. Finn, bmf2003@columbia.edu 
curacy of confidence judgments when participants, before rating their confidence in their answers, were asked to write down one reason contradicting the answers they had just given. Judgments showed a smaller overconfidence bias after participants generated and considered reasons why their answers could be wrong.

More recently, Koriat, Bjork, Sheffer, and Bar (2004) conducted an investigation that tested people's confidence in their memories across varying retention intervals. They tested whether people would give distinct judgments about how much they would recall on a test that came after a day, a week, or even a year. Predictions were vastly overconfident. Performance judgments about a test following a week delay were about the same as predictions about performance on a test immediately following study. However, when people were asked about how much they thought they would forget immediately, in a day, or in a week, judgments did show an effect of retention interval. As the retention interval increased, confidence about recall performance declined, as it should have. Forget judgments were sensitive to the retention interval, whereas remember judgments were not.

Both studies reported above suggest that reframing how a judgment is made can influence how people think about their memories and may serve to increase judgment accuracy. Because of the link between monitoring and control, study behavior may also improve. To date, the vast majority of the research on framing effects has focused on people's ethical- and economic-choice behavior. Research in these domains has demonstrated that, across a variety of tasks, people's judgments and choice preferences about an identical situation can vary as a function of whether the choice has been framed positively or negatively (Tversky \& Kahneman, 1981). In Tversky and Kahneman's (1981) famous "Asian Disease Problem," people are told that an outbreak of a disease in the United States is expected to kill 600 people. Participants are asked to choose between two programs to combat the disease. They are told that if Program A is used, 200 lives will be saved for sure, and that if Program B is used, there is a $1 / 3$ probability that 600 will be saved and a $2 / 3$ probability that no people will be saved. In this positive, gain frame, most choose Program A. However, when equivalent programs are described in terms of the number of people who will die (Program C, 400 will die for sure; Program D, 1/3 probability that no one will die, $2 / 3$ probability that all 600 will die) the majority of people choose Program D, despite the fact that $\mathrm{C}$ and $\mathrm{D}$ are simply reworded versions of $\mathrm{A}$ and $\mathrm{B}$. The only difference between the contrasting programs is that $\mathrm{A}$ and $\mathrm{B}$ are framed in terms of the number of lives that will be saved and C and D are framed in terms of the number of people who will die. Tversky and Kahneman (1981) described this finding as a shift from risk aversion and preference of an outcome that is certain when choices are framed in terms of gains to risk seeking when choices are framed in terms of loss.

A multitude of studies have demonstrated that framing effects have important implications for the kinds of social and economic decisions that people make (see Kühberger, 1997, for a review). Virtually no one (Koriat et al., 2004, excepted) has looked at the effect of framing on judgments about memory. The present research investigated the role of framing in metacognitive monitoring and control processes. In metacognition experiments, participants typically base judgments on whether they think they will remember each item on a later test. Of interest in the present study was whether framing the JOL in terms of forgetting would debias people's judgments about how well they had learned something, diminishing confidence and thus increasing the predictive accuracy about upcoming test performance.

The first research goal was to examine the effect of framing on immediate and delayed JOLs. Immediate JOLs taken after an initial study presentation were important judgments to investigate because they typically show a large overconfidence bias. In contrast, judgments taken at a delay are usually more accurate, show a truncated overconfidence bias, and are thought to rely on different heuristic information than do immediate judgments. A test of both types of JOLs allowed a focused characterization of the effect of framing on metacognitive monitoring.

The second research goal was to investigate the effects of framing on the control of learning. The question was whether framing effects would arise at the level of the study choice, both with respect to the number of items and to the relative ease of the items people would select for restudy. If the forget frame reduces confidence, study choices should also reflect that debiasing. One possible outcome of reduced confidence was that people would choose to restudy more overall and, in particular, select more of the easy items to restudy.

\section{EXPERIMENT 1A}

Experiment 1A contrasted immediate JOLs framed in terms of remembering and forgetting. In the remember condition, participants made typical JOLs in which they were asked to indicate how likely it was that they would remember each pair on the test coming up in a few minutes. In the forget condition, participants were asked how likely it was that they would forget each pair. The hypothesis was that when asked to make immediate JOLs within the forget frame, participants would be less confident than when JOLs were made within the remember frame.

\section{Method}

Participants, Design, and Materials. The participants were 48 undergraduates at Columbia University and Barnard College. They participated for course credit or cash. In this and in the experiments that follow, participants were treated in accordance with APA ethical guidelines. The experiment was a between-participants design. Participants were assigned randomly to either the remember or the forget condition. There were 24 participants in each condition.

Each participant studied 48 word pairs. The word lists were 48 cue-target word pairs composed of words taken from the Toronto Word Pool, a pool of 1,080 common English two-syllable words (Friendly, Franklin, Hoffman, \& Rubin, 1982). Mean word length of cue and target was 6.24 letters. No word exceeded 8 letters. For each participant, the computer randomly combined the words into pairs.

Procedure. Participants were instructed that they would be learning 48 word pairs, making judgments, and taking a cued recall test. At the beginning of the experiment, participants in the remember 
condition were given the typical JOL instructions asking them to make their judgments on the basis of what they thought the chances were that they would remember the second word when given the first word during a memory test that would occur in a few minutes. The forget frame instructions were identical, except that the word remember was replaced with the word forget. In both conditions, participants were asked to use a scale from $0 \%-100 \%$ to make their judgment. In the remember condition, participants were told to use numbers closer to $100 \%$ to indicate that they were sure that they would remember and to use numbers closer to $0 \%$ to indicate that they were sure that they would not. Participants in the forget condition were told to use numbers closer to $100 \%$ to indicate that they were sure that they would forget and to use numbers closer to $0 \%$ to indicate that they were sure that they would not. They were told that at test they would be given the cue and would have to type in the target.

Pairs were presented once, for $3.5 \mathrm{sec}$, and were immediately followed by a prompt to make the JOL. In the remember condition, participants were asked to provide their judgment of remembering, and in the forget condition, they were asked their judgment of forgetting, each time they were prompted to make a JOL. After all of the pairs had been studied and given judgments, the pairs were reshuffled and the participants were tested. Each cue was presented, and participants were asked to type in the target. There were no restrictions on the amount of time that they could spend on the test.

\section{Results}

Recall performance. Recall performance was not expected to differ between the two conditions. Recall performance means were $.17(S E=.02)$ for the remember condition and $.19(S E=.03)$ for the forget condition. The two conditions were not significantly different from one another $(t<1, p>.05)$, as evidenced by an independent samples $t$ test. A probability level of $p<.05$ was used as the criterion for statistical significance throughout.

JOLs. In this and in the experiments that follow, forget condition judgments were calculated as 1-judgment value so that the remember and the forget conditions could be compared on the same scale. As can be seen in Figure 1, judgments were significantly higher in the remember condition $(M=.51, S E=.04)$ than in the forget condition $(M=.37, S E=.03)$, by a difference of $.14[t(46)=2.83$, $\left.p<.05, \mathrm{CI}_{.95}=.04, .23\right]$. This result provided the first sign that framing effects occur with immediate JOLs.

A further analysis of the JOLs revealed significant differences between the remember and forget conditions in the number of items given low JOLs and the number of items given high JOLs. In this analysis, a judgment of less than 50 was classified as a low JOL, and a judgment of 50 or higher was classified as a high JOL. People in the forget condition made a greater number of low JOLs $(M=$ $35.50, S E=1.83)$ than did people in the remember condition $(M=26.63, S E=2.71)[t(46)=2.71, p<.05$, $\left.\mathrm{CI}_{.95}=2.28,15.47\right]$.

Calibration. An overconfidence bias was assessed by measuring calibration. A calibration score was calculated for each participant by subtracting mean recall performance from the mean judgment for each condition. Overconfidence was obtained if a positive score was significantly different from zero. Of interest was whether the forget judgments would be more calibrated (i.e., less overconfident) than the remember judgments. Participants in the remember condition were significantly more overcon-

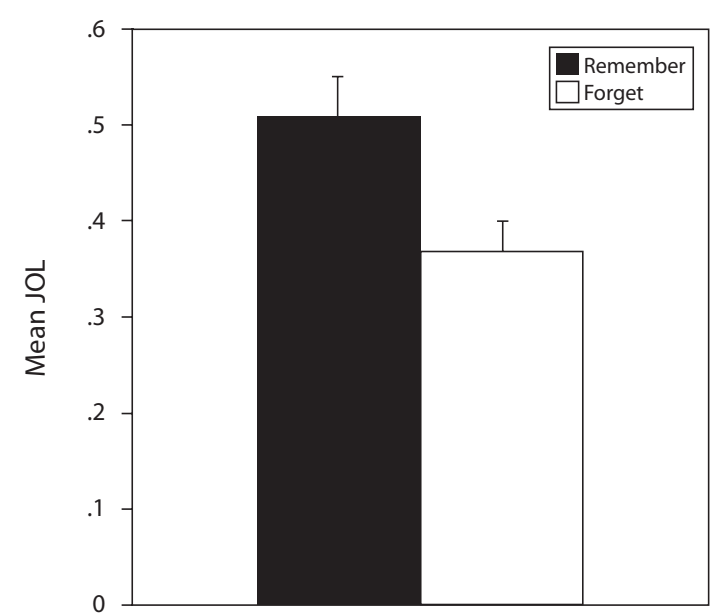

Figure 1. Mean JOLs for remember and forget conditions in Experiment 1.

fident $(M=.34, S E=.04)$ than those in the forget condition $(M=.19, S E=.03)\left[t(46)=3.07, p<.05, \mathrm{CI}_{.95}=\right.$ $.05, .25]$. Both were significantly different from zero (all $t \mathrm{~s}>1$, all $\mathrm{ps}<.05)$.

Gammas. For comprehensiveness, mean gamma correlations computed using each participant's gamma are reported for all three experiments in Table 1. Gammas are also given between JOLs and restudy choice for Experiments 2 and 3. Gamma correlations are a nonparametric statistic indicating predictive metacognitive accuracy of the JOLs with respect to recall or restudy choice. This accuracy measure is also called resolution or relative accuracy. These data indicate that in all cases, as measured by independent samples $t$ tests, there were no differences in relative accuracy between the remember and forget conditions.

\section{Discussion}

The results of Experiment 1A show that framing effects occur when people make immediate JOLs. Whereas recall performance did not differ between the two conditions, judgments framed in terms of forgetting were less confident, and less overconfident, than the remember frame judgments. The only methodological difference between the two conditions was the substitution of one word, for-

Table 1 Gamma Correlations

\begin{tabular}{|c|c|c|c|c|c|c|c|c|}
\hline & \multicolumn{4}{|c|}{ JOL-Recall } & \multicolumn{4}{|c|}{ JOL-Study Choice } \\
\hline & \multicolumn{2}{|c|}{ Remember } & \multicolumn{2}{|c|}{ Forget } & \multicolumn{2}{|c|}{ Remember } & \multicolumn{2}{|c|}{ Forget } \\
\hline & $M$ & $\overline{S E}$ & $M$ & $\overline{S E}$ & $M$ & $\overline{S E}$ & $M$ & $\overline{S E}$ \\
\hline Expe & .17 & .10 & $.42^{*}$ & .07 & - & - & - & - \\
\hline Experiment 1B & $.91^{*}$ & .07 & $.75^{*}$ & .11 & - & - & - & - \\
\hline Experiment 2 & $.31^{*}$ & .10 & .21 & .10 & $-.71^{*}$ & .10 & $-.51^{*}$ & .16 \\
\hline Experiment 3 & $.79^{*}$ & .02 & $.52^{*}$ & .13 & $-.76^{*}$ & .11 & $-.56^{*}$ & .14 \\
\hline
\end{tabular}

Note-Experiment 1A shows effects of immediate JOLs and Experiment $1 \mathrm{~B}$ shows effects of delayed JOLs. *Significantly different from zero. No significant differences between conditions were found for JOLrecall gammas or JOL-study choice gammas in any experiment (all $p \mathrm{~s}>$ $.05)$. 
get, for the word remember in the judgment instructions. This alone was enough to significantly reduce, though not eliminate, the persistent overconfidence bias shown with single study-test trial immediate JOLs.

JOLs made immediately after a study presentation are typically less accurate than those made after even a short delay (Dunlosky \& Nelson, 1992, 1994; Nelson \& Dunlosky, 1991). This accuracy advantage is thought to be due to a difference in the types of cues used to make the judgment. Immediate JOLs are thought to be based on a range of cues, including information in short-term memory (Nelson \& Dunlosky, 1991), normative ease (Koriat, 1997), or ease of encoding (Begg, Duft, Lalonde, Melnick, \& Sanvito, 1989; Hertzog, Dunlosky, Robinson, \& Kidder, 2003; Koriat \& Ma'ayan, 2005). In contrast, delayed JOLs typically involve a retrieval attempt, yielding a more accurate assessment of later eventual recall. This difference in cue utilization between immediate and delayed JOLs may modulate metacognitive biases, such as overconfidence (delayed JOLs show less overconfidence) and the underconfidence with practice effect (see Finn \& Metcalfe, 2007, 2008). For example, immediate JOLs show underconfidence on and after a second study-judgment-test trial (Koriat et al., 2002), whereas delayed JOLs typically do not (Koriat \& Ma'ayan, 2005; Koriat, Ma'ayan, Sheffer, \& Bjork, 2006; Meeter \& Nelson, 2003; Scheck \& Nelson, 2005; Serra \& Dunlosky, 2005). According to Finn and Metcalfe $(2007,2008)$, this is because immediate JOLs are not made on the basis of a target retrieval and instead rely on other, less diagnostic information, such as memory for performance on the prior test, which produces underconfident second-trial judgments. The approach adopted in Experiment 1B was to test whether the framing effect would generalize to delayed JOLs. The hypothesis was that framing effects would not arise in the case of delayed judgments, which have been shown to be less susceptible to confidence biases than immediate judgments are.

\section{EXPERIMENT 1B}

\section{Method}

Participants, Design, and Materials. The participants were 40 undergraduates at Columbia University and Barnard College. They participated for course credit or cash. There were 20 participants in each condition.

The experiment was identical to Experiment 1A, except that JOLs were made at a delay rather than immediately after study. After study, the word pairs were reshuffled and the cue was presented for a delayed JOL. After making delayed JOLs for each of the cues, the word pairs were reshuffled and the cue was presented for test.

\section{Results}

Recall performance. Mean recall performance for the remember condition was $.11(S E=.03)$. The forget condition had a mean recall performance of $12(S E=.03)$. The two conditions were not significantly different from one another $(t<1, p>.05)$.

JOLs. In contrast to immediate JOLs, delayed judgments were not significantly different in the remember and forget conditions $(t<1, p>.05)$. The mean JOL was $.24(S E=.03)$ in the remember condition and $.29(S E=$
$.04)$ in the forget condition. An analysis of the number of items given low JOLs revealed no significant differences $[t(38)=1.46, p>.05]$ between the remember $(M=$ $29.60, S E=1.51)$ and forget $(M=26.15, S E=1.83)$ conditions.

Calibration. There were no significant differences in calibration for the remember and forget conditions $(t<1$, $p>.05)$. The remember frame showed an overconfidence bias of $.14(S E=.03)$, which was significantly different from zero $\left[t(19)=5.11, p<.05, \mathrm{CI}_{.95}=.08, .20\right]$. The forget overconfidence bias was $.17(S E=.04)$, also significantly different from zero $[t(19)=3.98, p<.05$, $\left.\mathrm{CI}_{.95}=.08, .25\right]$.

\section{Discussion}

The results of Experiment 1B indicated that framing effects did not occur with delayed JOLs. In Experiment 1A, forget framed immediate JOLs were less confident than remember framed JOLs. Remember and forget framed delayed JOLs were not different. Whereas forget framed immediate JOLs showed a reduced overconfidence bias and a larger number of low JOLs in comparison with remember frame JOLs, no differences emerged when the judgments were delayed. To explore how framing effects influence restudy, the subsequent experiments focused on immediate judgments.

\section{EXPERIMENT 2}

An important implication of the findings in Experiment $1 \mathrm{~A}$ was that restudy choices, because of the link between judgments and study control, should differ depending on the frame. Overconfidence during restudy selection may be particularly insidious because overconfident people would be expected to study less than they need to. The first objective of Experiment 2 was to replicate the results of Experiment 1A by asking people to make either remember or forget framed JOLs. The second objective was to replicate the findings of Metcalfe and Finn (2008) and show that the study choices that immediately followed the judgments also reflected judgment differences driven by the framing effect. The specific prediction was that reduced confidence in the forget frame would lead to the selection of more items for restudy.

\section{Method}

Participants. The participants were 46 undergraduates at Columbia University and Barnard College. They received course credit or cash for participation. There were 22 participants in the remember condition and 24 participants in the forget condition.

Materials, Design, and Procedure. Experiment 2 used the same materials and design as those for Experiment 1A, except that the word list was shortened to 36 items and participants made restudy choices. After each study presentation, participants made a JOL. Instructions for the JOLs followed the same procedure in Experiment 1A; participants were instructed to make their judgments of remembering or of forgetting. After making each judgment, they were asked whether they would like to restudy that pair before the test. They made their study choices by hitting a "yes" or "no" button. Study choice framing always matched the judgment frame. In the remember frame, participants were prompted to make each study choice with the instructions "Would you like to restudy this pair to 
help you remember it before the test?" In the forget frame, participants were prompted with the instructions "Would you like to restudy this pair so that you don't forget it before the test?" After their study choice, they proceeded to the next item for study, JOL, and choice. After all of the words had been viewed through this procedure, the pairs were reshuffled for test. Participants were not allowed to restudy any items. At test, each cue was presented and participants were asked to provide the corresponding target.

\section{Results}

Recall performance. The recall performance mean for the remember condition was $.37(S E=.06)$. The mean for the forget condition was $.40(S E=.04)$. The difference in recall performance was not significant $(t<1, p>.05)$.

JOLs. The remember frame JOL mean was .56 ( $S E=$ $.04)$. The forget frame JOL mean was $.46(S E=.02)$, a difference that was significant $[t(44)=2.61, p<.05$, $\left.\mathrm{CI}_{95}=.02, .18\right]$ and is shown in Figure 2. As in Experiment $1 \mathrm{~A}$, participants in the forget condition made a greater number of low JOLs $(M=17.79, S E=1.79)$ than did people in the remember condition $(M=12.64, S E=$ $1.88)[t(44)=1.99, p=.05]$. These results replicated the findings in Experiment $1 \mathrm{~A}$ and provided additional evidence that a forget frame leads to the generation of lower immediate JOLs.

Calibration. There was a significant difference in calibration $\left[t(44)=2.07, p=.04, \mathrm{CI}_{.95}=.003, .25\right]$, with the remember condition showing significantly more overconfidence $(M=.19, S E=.05)$ than the forget condition. Participants in the remember condition were significantly overconfident $\left[t(21)=3.64, p<.05, \mathrm{CI}_{.95}=.08, .30\right]$, whereas participants in the forget condition were calibrated $(M=.06, S E=.03)$, as indexed by a nonsignificant difference from zero $[t(23)=1.83, p>.05]$.

Restudy choices. As can be seen in Figure 3, significantly fewer items were selected for restudy in the remember condition $(M=.45, S E=.07)$ than in the forget condition $(M=.65, S E=.06)$ by a difference of $.20[t(44)=$ $\left.2.22, p<.05, \mathrm{CI}_{.95}=.02, .39\right]$. This large and significant

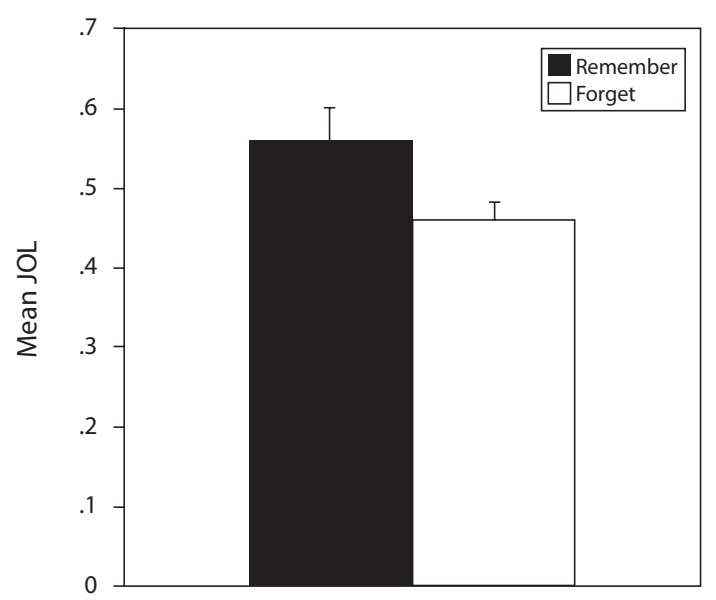

Figure 2. Mean JOLs for remember and forget conditions in Experiment 2.



Figure 3. Proportion of items chosen for restudy for remember and forget conditions in Experiment 2.

difference indicated that framing effects can alter people's study behavior. ${ }^{1}$

A further question was whether the ease of the items selected for restudy would change as people became less confident. One possible pattern of results was that, as everything was perceived as more difficult within the forget frame, people would equally increase the number of easy, medium, and difficult items for restudy. Another potential pattern was that, when people's confidence was lower, their study choices would shift to include more of the easiest items. When people are overconfident, few of the easiest items are likely to be selected for restudy. As confidence shifts downward, the number of easy items people feel they should study is likely to increase, whereas the number of difficult items selected for restudy might remain stable across the remember and forget frames, or might even decrease. Individuals' JOLs were used to determine which items were easy, medium, or difficult because no a priori measures of objective difficulty were taken. For each participant, JOLs ranging between $0 \%$ and $33 \%$ were labeled as "difficult," 34\%-66\% were labeled as "medium," and $67 \%-100 \%$ were labeled as "easy." Study choice results were analyzed using a 2 (remember or forget frame) $\times 3$ (easy, medium, or difficult) repeated measures ANOVA, with vocabulary ease as a within-participants factor and judgment frame as a between-participants factor. There was a main effect of ease $\left[F(2,74)=22.57, M S_{\mathrm{e}}=.07\right.$, $\left.p<.05 ; \eta_{\mathrm{p}}^{2}=.38\right]$. People chose to study difficult items the most $(M=.73, S E=.06)$, followed by medium items $(M=.57, S E=.06)$ and easy items $(M=.31, S E=.06)$. There was also a main effect of frame condition $[F(2,37)=$ $\left.6.73, M S_{\mathrm{e}}=.24, p<.05 ; \eta_{\mathrm{p}}^{2}=.15\right]$. As reported earlier, more items were chosen within the forget frame than within the remember frame. The interaction between ease and frame condition was not significant $[F(2,74)=0.88$, $\left.M S_{\mathrm{e}}=.07, p>.05\right]$; however, because of an interest in how the ease of items selected for restudy would change or remain stable across frames, additional comparisons at each of the vocabulary ease levels were conducted. Significant differences emerged in the mean number of easy items selected for restudy (remember, $M=.19, S E=.07$; forget, $M=.48, S E=.09)\left[t(37)=2.82, p<.05, \mathrm{CI}_{.95}=\right.$ $.09, .57]$. There were no significant differences between the conditions in the number of medium items $[t(37)=$ 
$1.63, p>.05]$ or difficult items $[t(37)=1.63, p>.05]$ selected for restudy.

\section{Discussion}

A consistent pattern emerged across Experiments 1A and 2. Forget framing reduced confidence and prompted people to select more items for restudy. Experiment 2 extended the findings of Experiment 1A by showing that the remember condition, the condition that demonstrated greater overconfidence, was also the condition in which fewer items were selected for restudy. An analysis that used people's JOLs as an indication of which items were easy, medium, or difficult hinted that, as people became less confident, the number of items that seemed difficult increased. People in the forget condition chose more items that were easy to restudy than did people in the remember condition, suggesting that as confidence shifted downward, people modified the control of their learning to include more of the easiest items, in particular.

To investigate more thoroughly how people made study choices across a range of difficulty, item ease was manipulated systematically in Experiment 3. In Experiment 3 , participants were asked to study easy, medium, and difficult Spanish-English vocabulary pairs and make judgments and study choices within either a remember or a forget frame.

\section{EXPERIMENT 3}

\section{Method}

Participants. The participants were 42 undergraduates at Columbia University and Barnard College. They received course credit or cash for participation. None of the participants were native Spanish speakers. There were 21 participants in each condition.

Materials, Design, and Procedure. The materials were SpanishEnglish vocabulary pairs. There were 12 pairs in each of the three conditions (easy, medium, and difficult), for a total of 36 pairs. The vocabulary pairs came from Metcalfe (2002) and had been created to encompass three difficulty conditions. Participant recall performance in the four experiments in Metcalfe (2002) established these difficulty levels. The experiment was a mixed design with vocabulary ease as a within-participants factor and framing condition as a between-participants factor. Participants were assigned randomly to either the remember or the forget condition, and all participants studied easy, medium, and difficult pairs. With the exception of the materials, the procedure was identical to that in Experiment 2. All 36 pairs were shuffled and presented for $3 \mathrm{sec}$ of study. Immediately following the presentation of each pair, participants made a remember or forget frame judgment followed by a restudy choice. Word pairs were shuffled by the computer before the start of the test. At test, the Spanish word was presented and participants were asked to type in the English translation. When they had entered in their answer, they hit "Return" and the next Spanish cue appeared. There were no constraints on the time they could spend on each item at test.

\section{Results}

Recall performance. There was a main effect of ease $\left[F(2,80)=141.88, M S_{\mathrm{e}}=.03, p<.05 ; \eta_{\mathrm{p}}^{2}=.78\right]$. Recall performance was best for the easy items $(M=.98, S E=$ $.01)$, followed by the medium items $(M=.68, S E=.04)$ and the difficult items $(M=.39, S E=.04)$. All ease conditions were significantly different from one another (all $t \mathrm{~s}>1, p \mathrm{~s}<.05)$. No other main effect or interaction was significant.

JOLs. Mean judgments for remember and forget conditions at each level of difficulty are plotted in Figure 4. There was a main effect of ease $\left[F(2,80)=232.09, M S_{\mathrm{e}}=\right.$ $\left..01, p<.05 ; \eta_{\mathrm{p}}^{2}=.85\right]$. Easy items were given the highest JOLs $(M=.94, S E=.01)$, followed by medium items $(M=.64, S E=.03)$ and difficult items $(M=.38, S E=$ $.04)$ (all $t \mathrm{~s}>1, p \mathrm{~s}<.05)$. There was also a main effect of condition $\left[F(2,40)=4.61, M S_{\mathrm{e}}=.07, p<.05 ; \eta_{\mathrm{p}}^{2}=.10\right]$. As in Experiments 1A and 2, people in the remember condition made higher JOLs overall $(M=.70, S E=.03)$ than did people in the forget condition $(M=.60, S E=.03)$. The interaction between item ease and frame condition was not significant. Planned comparisons revealed significant differences for the easy items (remember frame, $M=.97, S E=.01$; forget frame, $M=.91, S E=.02$ ) $[t(40)=2.26, p<.05]$ and medium items (remember frame, $M=.71, S E=.04$; forget frame, $M=.57, S E=$ $.04)[t(40)=2.28, p<.05]$, but no difference for the difficult items (remember frame, $M=.43, S E=.06$; forget frame, $M=.33, S E=.04)[t(40)=1.48, p>.05]$.

Calibration. There were no significant main effects of ease or condition on calibration, nor was the interaction between ease and frame condition significant. Calibration values for both groups across item ease did not differ significantly from zero (remember frame, $M=-.02$, $S E=.04, t<1, p>.05$; forget frame, $M=-.05, S E=$ $.04, t=1.34, p>.05)$. Planned comparisons revealed significant differences only for the easy items (remember frame, $M=-.02, S E=.01$; forget frame, $M=-.07$, $S E=.03)$. Remember frame calibration for the easy items was not different from zero $[t(20)=1.13, p>.05]$, but forget frame calibration was significantly underconfident $[t(20)=2.65, p<.05]$. Calibration for all of the other levels was not significantly different from zero.

Restudy choices. Restudy choices showed a main effect of ease $\left[F(2,80)=83.73, M S_{\mathrm{e}}=.01, p<.05 ; \eta_{\mathrm{p}}^{2}=\right.$ .68]. Across frame conditions, people selected to study difficult items the most $(M=.71, S E=.05)$, followed by medium items $(M=.49, S E=.05)$ and easy items $(M=$ $.12, S E=.03)$. There was also a main effect of condition



Figure 4. Mean JOLs for remember and forget conditions at each of easy, medium, and difficult levels of vocabulary difficulty in Experiment 3. 
$\left[F(2,40)=5.11, M S_{\mathrm{e}}=.17, p<.05 ; \eta_{\mathrm{p}}^{2}=.11\right]$. This replicated the findings in earlier experiments showing that people in the remember condition chose to restudy fewer items overall $(M=.36, S E=.05)$ than did people in the forget condition $(M=.52, S E=.05)$. The item ease $\times$ frame condition interaction was not significant. However, planned comparisons revealed that people in the forget condition chose to study more easy items $(M=.21, S E=$ .06) than did people in the remember condition $(M=.03$, $S E=.02)\left[t(29)=2.44, p<.05, \mathrm{CI}_{.95}=.08, .20\right]$. Indeed, the number of easy items selected in the remember frame condition did not significantly differ from zero $[t(20)=$ $1.66, p>.05]$. A comparison of the choices for medium difficulty also revealed a significant difference between the frame conditions $\left[t(40)=1.81, p<.05, \mathrm{CI}_{.95}=.10\right.$, .18]. People in the forget condition selected more medium difficulty items for restudy $(M=.62, S E=.07)$ than did people in the remember condition $(M=.44, S E=.06)$. Remember and forget frame did not show any difference in number of difficult items selected for restudy $(M=.69$, $S E=.08 ; M=.79, S E=.06$, accordingly; $t<1, p>.05)$. These results are plotted in Figure 5.

\section{Discussion}

Participants gave lower JOLs and chose to restudy more items overall in the forget than in the remember frame, despite identical recall performance. The forget frame led people to choose more easy and medium items to restudy in particular. The results also showed that JOLs and study choices were tightly linked. Choices followed the same pattern as did JOLs across the easy, medium, and difficult items in the two conditions. JOLs were lower for the easy and the medium items in the forget condition, and the easy and medium items were also selected for study more often in the forget condition. With difficult items, JOLs did not differ between the remember and forget conditions and neither did choices.

\section{GENERAL DISCUSSION}

The most important finding in the present experiments was that reframing immediate JOLs in terms of forgetting, in contrast to remembering, changes how people monitor and control their learning. The forget frame reduced

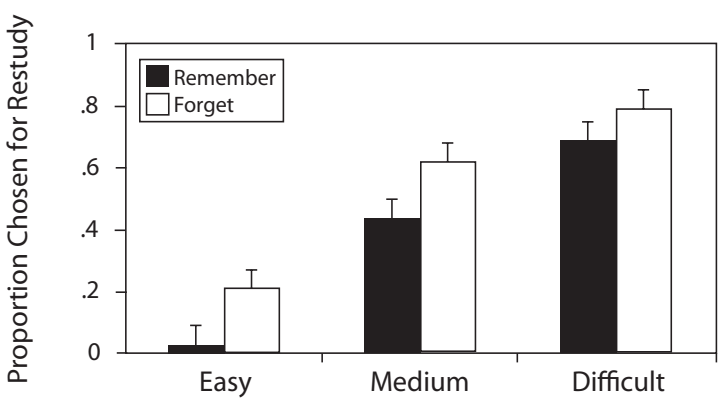

Figure 5. Proportion of items chosen for restudy for remember and forget conditions at each of easy, medium, and difficult levels of vocabulary difficulty in Experiment 3. confidence, resulting in judgments that showed a smaller overconfidence bias. In addition, the forget frame led to the selection of more items for restudy than did the remember frame.

In Experiments 2 and 3, people who were less confident chose to restudy the easiest items. This pattern is generally consistent with the region of proximal learning model of study time allocation (e.g., Metcalfe, 2002). The region of proximal learning model suggests that people should study items that are the closest to being learned. These are the items in the learner's region of proximal learning and are what Atkinson (1972a, 1972b) called "transitional items." As people's confidence dropped, so did their perception about what items could benefit most from additional study. The forget frame contributed to the number of items people perceived as difficult overall, but, as the region of proximal learning model would predict, they opted to increase the number of easy (Experiments 2 and 3) and medium (Experiment 3) items for restudy.

These experiments show clearly that framing effects have an impact on metamemory judgments and restudy choices. What are the processes that underlie these effects? One possibility is that the forget frame reduced confidence by making people more sensitive to the fallibility of their memories. According to Koriat et al. (2004, p. 654), "the mere mention of forgetting can activate people's knowledge about the decline in memory performance that is expected to occur with time." When people make a typical remember framed immediate JOL, they can potentially rely on several different types of cues (Begg et al., 1989; Hertzog et al., 2003; Koriat, 1997; Koriat \& Ma'ayan, 2005; Nelson \& Dunlosky, 1991). The results here suggest that a forget frame can prompt people to utilize additional information about how their memories may potentially fail and that making use of this cue yields judgments that are more accurate. Interestingly, these framing effects were confined to immediate JOLs. In Experiment 1B, delayed remember and forget frame JOLs were not different. These results indicate that when diagnostic information about whether the item can be retrieved from memory is available, as is the case when one is making a delayed JOL, other cues do not play as large a role in making the judgment.

Within the judgment and decision-making literature, there are a number of potential explanations for why framing effects occur (see Levin, Schneider, \& Gaeth, 1998, for a review). According to prospect theory, Kahneman and Tversky's (1979) widely accepted descriptive model of decision making, people are more sensitive to losses than they are to comparable gains; that is, losses "loom larger" than gains. Framing effects, whereby people - and capuchin monkeys for that matter (Chen, Lakshminaryanan, \& Santos, 2006) - respond differently depending on whether a choice or scenario is framed in terms of a gain or a loss, can occur because of loss aversion. The characterization of loss aversion corresponds well with the idea that the forget frame can activate cues about memory failure, producing a shift in people's metamemory judgments and restudy choices. When people were asked to evaluate their memories, the forget frame may have pointed toward potential loss of memory. This loss frame may have worked to shift 
people toward a more conservative criterion for endorsing something as learned, as well as to the selection of more, as well as easier, items to restudy in an effort to avoid the potential risk of losing those items from memory.

People who do not exhibit overconfidence have been shown to perform better on a subsequent test (Maki, Shields, Wheeler, \& Zachilli, 2005). It may be that successful learners are better at making metacognitive predictions, but the demonstrated link between monitoring accuracy and test performance has been mixed. Several studies have shown a positive correlation between metacognitive ability and test performance (Bisanz, Vesonder, \& Voss, 1978; Maki \& Berry, 1984; Yan, 1994), whereas others have shown that learning ability is not related to metacognitive accuracy (Kearney \& Zechmeister, 1989; Kelemen, Frost, \& Weaver, 2000; Lovelace, 1984; Maki \& Swett, 1987; Underwood, 1966). The present results support the proposal that lower confidence may change how people evaluate and plan their study, which may play an important role in later test accuracy. Performance may improve for people who are less confident, because they are restudying items that benefit most from additional study, in particular the easy and medium items. Another possible outcome is that lower confidence influences the amount of time people initially spend trying to learn the word. These possibilities are currently under investigation.

These results are important for several reasons. Overconfidence is a persistent problem with immediate JOLs. Simply framing immediate judgments in terms for forgetting reduced the overconfidence bias underscoring the importance of absolute accuracy for study choice behavior. The present results, therefore, are not only exciting because of the finding that a word can significantly change how people think about their memories, but more importantly because of the influence that such a change can have on improving people's choices about their learning behavior.

\section{AUTHOR NOTE}

This research was supported by NIMH Grant R01 MH60637. The author thanks the MetaLab as well as Lisa Son and Nate Kornell for their help and comments. Correspondence should be addressed to B. Finn, Department of Psychology, Columbia University, New York, NY 10027 (e-mail: bmf2003@columbia.edu).

\section{REFERENCES}

AtKInson, R. C. (1972a). Ingredients for a theory of instruction. American Psychologist, 27, 921-931.

AtKinson, R. C. (1972b). Optimizing the learning of a second-language vocabulary. Journal of Experimental Psychology, 96, 124-129.

Begg, I., Duft, S., Lalonde, P., Melnick, R., \& Sanvito, J. (1989). Memory predictions are based on ease of processing. Journal of Memory \& Language, 28, 610-632.

Benjamin, A. S., Bjork, R. A., \& Schwartz, B. L. (1998). The mismeasure of memory: When retrieval fluency is misleading as a metamnemonic index. Journal of Experimental Psychology: General, 127, 55-68.

Bisanz, G. L., Vesonder, G. T., \& Voss, J. F. (1978). Knowledge of one's own responding and the relation of such knowledge to learning: A developmental study. Journal of Experimental Child Psychology, 25, 116-128.

Chen, M. K., Lakshminaryanan, V., \& Santos, L. R. (2006). The evolution of our preferences: Evidence from capuchin monkey trading behavior. Journal of Political Economy, 114, 517-537.
Dunlosky, J., \& Hertzog, C. (1998). Training programs to improve learning in later adulthood: Helping older adults educate themselves. In D. J. Hacker, J. Dunlosky, \& A. C. Graesser (Eds.), Metacognition in educational theory and practice (pp. 249-275). Mahwah, NJ: Erlbaum.

Dunlosky, J., \& Nelson, T. O. (1992). Importance of the kind of cue for judgments of learning (JOL) and the delayed-JOL effect. Memory \& Cognition, 20, 374-380.

Dunlosky, J., \& Nelson, T. O. (1994). Does the sensitivity of JOLs to various study activities depend on when the JOLs occur? Journal of Memory \& Language, 33, 545-565.

Finn, B., \& Metcalfe, J. (2007). The role of memory for past test in the underconfidence with practice effect. Journal of Experimental Psychology: Learning, Memory, \& Cognition, 33, 238-244.

FinN, B., \& Metcalfe, J. (2008). Judgments of learning are influenced by memory for past test. Journal of Memory \& Language, 58, 19-34.

FischHoff, B., Slovic, P., \& Lichtenstein, S. (1977). Knowing with certainty: The appropriateness of extreme confidence. Journal of Experimental Psychology: Human Perception \& Performance, 3, 552-564.

Friendly, M., Franklin, P. E., Hoffman, D., \& Rubin, D. C. (1982). The Toronto Word Pool: Norms for imagery, concreteness, orthographic variables, and grammatical usage for 1,080 words. Behavior Research Methods \& Instrumentation, 14, 375-399.

Glenberg, A. M., Wilkinson, A. C., \& Epstein, W. (1982). The illusion of knowing: Failure in the self-assessment of comprehension. Memory \& Cognition, 10, 597-602.

Hertzog, C., Dunlosky, J., Robinson, A. E., \& Kidder, D. P. (2003). Encoding fluency is a cue used for judgments about learning. Journal of Experimental Psychology: Learning, Memory, \& Cognition, 29, 22-34.

Hirt, E. R., Kardes, F. R., \& Markman, K. D. (2004). Activating a mental simulation mind set through generation of alternatives: Implications for debiasing in related and unrelated domains. Journal of Experimental Social Psychology, 40, 374-383.

Hirt, E. R., \& Markman, K. D. (1995). Multiple explanation: A consider-an-alternative strategy for debiasing judgments. Journal of Personality \& Social Psychology, 69, 1069-1086.

HocH, S. J. (1985). Counterfactual reasoning and accuracy in predicting personal events. Journal of Experimental Psychology: Learning, Memory, \& Cognition, 11, 719-731.

Kahneman, D., \& Tversky, A. (1979). Prospect theory: An analysis of decisions under risk. Econometrica, 47, 313-327.

KeArney, E. M., \& Zechmeister, E. B. (1989). Judgments of item difficulty by good and poor associative learners. American Journal of Psychology, 102, 365-383.

Kelemen, W. L., Frost, P. J., \& Weaver, C. A., III (2000). Individual differences in metacognition: Evidence against a general metacognitive ability. Memory \& Cognition, 28, 92-107.

Koenler, D. J. (1991). Explanation, imagination, and confidence in judgment. Psychological Bulletin, 110, 499-519.

Koriat, A. (1993). How do we know what we know? The accessibility model of feeling of knowing. Psychological Review, 100, 609-639.

Koriat, A. (1997). Monitoring one's knowledge during study: A cueutilization approach to judgments of learning. Journal of Experimental Psychology: General, 126, 349-370.

Koriat, A. (2002). Metacognition research: An interim report. In T. J. Perfect \& B. L. Schwartz (Eds.), Applied metacognition (pp. 261-286). Cambridge: Cambridge University Press.

KorIAT, A., \& BJORK, R. A. (2006). Mending metacognitive illusions: A comparison of mnemonic-based and theory-based procedures. Journal of Experimental Psychology: Learning, Memory, \& Cognition, 32, 1133-1145.

Koriat, A., BJork, R. A., Sheffer, L., \& Bar, S. K. (2004). Predicting one's own forgetting: The role of experience-based and theorybased processes. Journal of Experimental Psychology: General, 133, 643-656.

Koriat, A., Lichtenstein, S., \& Fischhoff, B. (1980). Reasons for confidence. Journal of Experimental Psychology: Human Learning \& Memory, 6, 107-118.

Koriat, A., \& MA'AYAn, H. (2005). The effects of encoding fluency and retrieval fluency on judgments of learning. Journal of Memory \& Language, 52, 478-492. 
Koriat, A., Ma'ayan, H., Sheffer, L., \& BJork, R. A. (2006). Exploring a mnemonic debiasing account of the underconfidence-withpractice effect. Journal of Experimental Psychology: Learning, Memory, \& Cognition, 32, 595-568.

Koriat, A., Sheffer, L., \& Ma'Ayan, H. (2002). Comparing objective and subjective learning curves: Judgments of learning exhibit increased underconfidence with practice. Journal of Experimental Psychology: General, 131, 147-162.

Kornell, N., Son, L. K., \& Terrace, H. S. (2007). Transfer of metacognitive skills and hint seeking in monkeys. Psychological Science, 18, 64-71.

KÜHBERGER, A. (1997). Theoretical conceptions of framing effects in risky decisions. In R. Ranyard, W. R. Crozier, \& O. Svenson (Eds.), Decision making: Cognitive models and explanations (pp. 128-144). New York: Routledge.

LeVIN, I. P., Schneider, S. L., \& GAeth, G. J. (1998). All frames are not created equal: A typology and critical analysis of framing effects. Organizational Behavior \& Human Decision Processes, 76, 149-188.

Lichtenstein, S., \& FischHoff, B. (1980). Training for calibration. Organizational Behavior \& Human Performance, 26, 149-171.

Lichtenstein, S., FischHoff, B., \& Phillips, L. D. (1982). Calibration of probabilities: The state of the art to 1980. In D. Kahneman, P. Slovic, \& A. Tversky (Eds.), Judgment under uncertainty: Heuristics and biases (pp. 306-334). New York: Cambridge University Press.

Lovelace, E. A. (1984). Metamemory: Monitoring future recallability during study. Journal of Experimental Psychology: Learning, Memory, \& Cognition, 10, 756-766.

MAKI, R. H. (1998). Predicting performance on text: Delayed versus immediate predictions and tests. Memory \& Cognition, 26, 959-964.

MaKI, R. H., \& Berry, S. L. (1984). Metacomprehension of text material. Journal of Experimental Psychology: Learning, Memory, \& Cognition, 10, 663-679.

MaKi, R. H., Shields, M., Wheeler, A. E., \& Zacchilli, T. L. (2005). Individual differences in absolute and relative metacomprehension accuracy. Journal of Educational Psychology, 97, 723-731.

MAKI, R. H., \& SWETT, S. (1987). Metamemory for narrative text. Memory \& Cognition, 15, 72-83.

Mazzoni, G., \& CoRnoldi, C. (1993). Strategies in study time allocation: Why is study time sometimes not effective? Journal of Experimental Psychology: General, 122, 47-60.

Meeter, M., \& Nelson, T. O. (2003). Multiple study trials and judgments of learning. Acta Psychologica, 113, 123-132.

Metcalfe, J. (1998). Cognitive optimism: Self-deception or memorybased processing heuristics? Personality \& Social Psychology Review, 2, 100-110.

MetCalfe, J. (2002). Is study time allocated selectively to a region of proximal learning? Journal of Experimental Psychology: General, 131, 349-363.

Metcalfe, J., \& FinN, B. (2008). Evidence that judgments of learning are causally related to study choice. Psychonomic Bulletin \& Review, 15, 174-179.

Metcalfe, J., Schwartz, B. L., \& Johquim, S. G. (1993). The cuefamiliarity heuristic in metacognition. Journal of Experimental Psychology: Learning, Memory, \& Cognition, 19, 851-861.

Nelson, T. O., \& Dunlosky, J. (1991). When people's judgments of learning (JOLs) are extremely accurate at predicting subsequent recall: The "delayed-JOL effect." Psychological Science, 2, 267-270.
Nelson, T. O., \& Narens, L. (1990). Metamemory: A theoretical framework and new findings. In G. H. Bower (Ed.), The psychology of learning and motivation (Vol. 26, pp. 125-141). New York: Academic Press.

Pressley, M., \& Ghatala, E. S. (1990). Self-regulated learning: Monitoring learning from text. Educational Psychologist, 25, 19-33.

REDER, L. M. (1987). Strategy selection in question answering. Cognitive Psychology, 19, 90-138.

REDER, L. M. (1988). Strategic control of retrieval strategies. In G. H. Bower (Ed.), The psychology of learning and motivation (Vol. 22, pp. 227-259). San Diego: Academic Press.

Scheck, P., \& Nelson, T. O. (2005). Lack of pervasiveness of the underconfidence-with-practice effect: Boundary conditions and an explanation via anchoring. Journal of Experimental Psychology: General, 134, 124-128.

SERRA, M. J., \& Dunlosky, J. (2005). Does retrieval fluency contribute to the underconfidence-with-practice effect? Journal of Experimental Psychology: Learning, Memory, \& Cognition, 31, 1258-1266.

Son, L. K., \& Metcalfe, J. (2005). Judgments of learning: Evidence for a two-stage process. Memory \& Cognition, 33, 1116-1129.

THIEDE, K.W. (1999). The importance of monitoring and self-regulation during multitrial learning. Psychonomic Bulletin \& Review, 6, 662-667.

Tversky, A., \& Kahneman, D. (1974). Judgment under uncertainty: Heuristics and biases. Science, 185, 1124-1130.

TVersky, A., \& Kahneman, D. (1981). The framing of decisions and the psychology of choice. Science, 211, 453-458.

UNDERWOOD, B. J. (1966). Individual and group predictions of item difficulty for free learning. Journal of Experimental Psychology, 71, 673-679.

YAN, W. (1994). Learning ability and memory monitoring. Intelligence, 18, 215-229.

Yates, J. F., Veinott, E. S., \& Patalano, A. L. (2003). Hard decisions, bad decisions: On decision quality and decision aiding. In S. L. Schneider \& J. Shanteau (Eds.), Emerging perspectives on judgment and decision research (pp. 13-63). New York: Cambridge University Press.

Zechmeister, E. B., \& Shaughnessy, J. J. (1980). When you know that you know and when you think that you know but you don't. Bulletin of the Psychonomic Society, 15, 41-44.

\section{NOTE}

1. It is generally agreed that when people make choices about restudying, they are doing so after having assessed the current learned state of the item they are evaluating, with or without an explicit judgment phase (Nelson \& Narens, 1990; Son \& Metcalfe, 2005; and see Kornell, Son, $\&$ Terrace, 2007, for hint-taking results with monkeys). An additional experiment was conducted in which study choices were made without an immediately preceding judgment. Although recall performance for the remember and forget conditions was not different $[t(46)=1.20, p>.05]$, restudy choices did show a .23 difference $\left[t(46)=2.70, p<.05, \mathrm{CI}_{95}=\right.$ $.06, .40]$. Even without an explicit JOL phase, forget framing led to the selection of more items (.62) than in the remember condition (.39).

(Manuscript received June 27, 2007;

revision accepted for publication November 25, 2007.) 